\title{
Fleißig? Genial?
}

$\mathrm{B}$ ei Schulprüfungen lässt sich regelmäßig beobachten, dass es offenbar zwei grundsätzlich unterschiedliche Herangehensweisen an eine osteopathische Ausbildung oder sogar an die Osteopathie an sich gibt: Zum einen sind da die Fleißigen, die den gesamten Lernstoff auswendig können, immer eine passende Antwort wissen und jede Technik genau so demonstrieren, wie sie im Unterricht besprochen wurde. Gegebenenfalls zeigen sie sogar, mit welchen Abweichungen die einzelnen Dozenten sie gezeigt haben. Anatomie, Biomechanik, Physiologie und Embryologie stellen für sie die Leitschnur dar zur Findung einer Diagnose und Entwicklung einer Behandlungsstrategie. Da sich meist auch die Dozenten aus der Gruppe der Fleißigen rekrutieren, entwickelt sich schnell ein Gefühl der Harmonie. Man kann sich aufeinander verlassen. Es passiert nichts Unvorhergesehenes. Auch schwere Fragen werden unter anerkennendem Nicken bravourös gemeistert.

Mit dieser Harmonie ist es schnell vorbei, wenn dann Prüflinge aus der zweiten Gruppe antreten: die der Genialen. Im richtigen Leben lassen sie sich von den Geweben führen und wissen immer intuitiv, was jeder einzelne Patient benötigt. Deshalb belasten sie sich weniger gerne mit überflüssigem Wissensgepäck. Mit den im Unterricht vermittelten Techniken wird sofort unbefangen gespielt, werden Variationen ausprobiert und siehe da: sie funktionieren ja auch in völlig anderem Kontext wunderbar. Der unangenehme Teil - und man weiß es schon vorher - sind die Prüfungen. Leider hat dann der Proband, an dem man demonstrieren soll („Stellen Sie sich vor, Ihr Patient hat...") nicht wirklich dieses Problem. Es gibt keine Gewebe, die einen führen, und während man bei echten Patienten intuitiv weiß, was zu tun ist, werden jetzt reine Techniken gefordert („Wie ging denn das noch mal...?“). Unsicherheit tritt auf - auch bei den Prüfern („Eigentlich konnte die/der doch mehr!?“).

Und so besteht die Gefahr, dass die Genialen ungerechtfertigterweise einer dritten Gruppe zugerechnet werden: derjenigen, die weder fleißig noch mit großen intuitiven Fähigkeiten ausgestattet sind. Aus dieser zahlenmäßig meist eher kleinen Gruppe rekrutieren sich nämlich diejenigen, denen das Bestehen der Prüfung verweigert werden muss.

Da stehen dann die Genialen, die ihre Patienten immer so wundervoll osteopathisch behandeln, und bangen um das Bestehen der Leistungskontrolle. Denn natürlich müssen Mindeststandards eingefordert werden. Die Prüfung soll schließlich das Ausbildungsniveau einer Schule widerspiegeln.

Offensichtlich bedeuten mäßig gute Prüfungsergebnisse bei Einzelnen also nicht unbedingt, dass es sich um schlechte Osteopathen handelt, die keinesfalls Lehrer werden sollten. Und was machen die Fleißigen, wenn ihre Patienten nicht der Norm entsprechen, das Gelernte sie nicht mehr trägt? Die moralische Schlussfolgerung muss jetzt unbedingt lauten, dass sich Fleiß und Genialität im Idealfall optimal ergänzen. Für die Patienten stellen solchermaßen Begnadete einen absoluten Glücksfall dar - aber was ist im Leben schon ideal?

Bei aller Schwarz-Weiß-Malerei: vielleicht trägt dieses Editorial ein wenig dazu bei, dass wir einander besser verstehen.

Die DO feiert ihr fünfjähriges Bestehen! Dafür danken wir unseren treuen Lesern und engagierten Autoren. Wir wollen für Sie auch in Zukunft eine anspruchsvolle, serviceorientierte und aktuelle Osteopathie-Zeitschrift herausgeben. Deshalb freuen wir uns auf weitere interessante Beiträge und konstruktive Kritik.

\section{Die Herausgeber}

Titelbild: Lymphstämme (Trunci lymphatici) im Thorax; Lymphknoten im Thorax in der Ansicht von dorsal. Aus: Schünke M, Schulte E, Schumacher U: Prometheus LernAtlas der Anatomie. Hals und Innere Organe. Illustrationen von M. Voll und K. Wesker. Stuttgart: Thieme; 2005. Bearbeitung: Pdesign. Stuttgart 2007. 